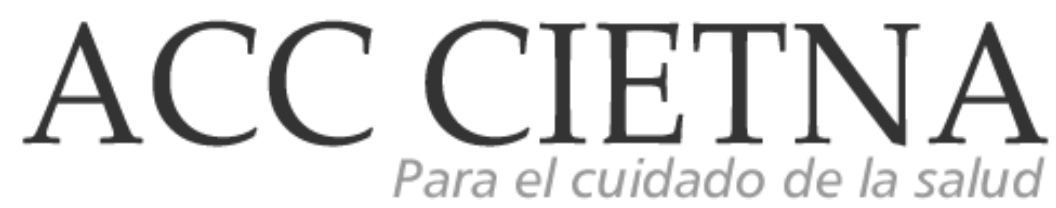

https:/ / doi.org/10.35383/cietna.v7i1.348

ENSAYO

\title{
Cuidados en el recién nacido de acuerdo a creencias y costumbres en diferentes culturas
}

\author{
Banda Pérez Antonieta de Jesús ', Álvarez Aguirre Alicia², Casique Casique Leticia ${ }^{3}$
}

\begin{tabular}{l}
\hline INFORMACIÓN DEL ENSAYO \\
\hline Historia del artículo: \\
Recibido el 10 de abril de 2020 \\
Aceptado el 10 de mayo de 2020 \\
\hline Palabras clave: \\
Cuidados \\
Cultura \\
Enfermería transcultural \\
Mujeres indígenas \\
Recién nacido
\end{tabular}

\section{RESUMEN}

El presente ensayo corresponde a una revisión sobre los cuidados culturales en el recién nacido en las mujeres indígenas y su aporte para la enfermería transcultural. En las zonas indígenas, el cuidado posee una cosmovisión de sus propias creencias, costumbres, tradiciones y normas relacionadas con la salud o el tratamiento de la enfermedad. En general, los estados con mayor presencia indígena presentan los niveles más altos de mortalidad materna. El conocer los cuidados culturales permite identificar y significar las prácticas y creencias que suelen pensarse como tradiciones retrógradas comparadas con el avance técnico científico al establecer un puente de comunicación entre los cuidados propios que tienen las mujeres indígenas. Se concluye en concreto el nacimiento de un niño es considerado un acontecimiento de gran importancia social y cultural no sólo para la familia, sino también para la comunidad. Se puede decir que es necesario alcanzar competencia cultural para ofrecer cuidado culturalmente congruente a las necesidades de estas mujeres en cuanto a los cuidados en el recién nacido. Es indispensable obtener conocimientos por medio de la investigación, llevar los productos de la investigación a la práctica del cuidado de enfermería y así avanzar hacia la enfermería transcultural.

\footnotetext{
1Estudiante de Doctorado en Ciencias de Enfermería, Campus Celaya Salvatierra Universidad de Guanajuato. Docente de la Facultad de Enfermería y Nutrición de la Universidad Autónoma de San Luis Potosí, México. E-mail: antonieta.banda@uaslp.mx. ORCID: https://orcid.org/0000-0001-95860641

2Doctora en Ciencias de Enfermería. Docente en Campus Celaya Salvatierra. División de Ciencias de la Salud e Ingenierías Departamento de Enfermería Clínica de la Universidad de Guanajuato, México. Teléfono: 052 (461) 5985922. E-mail alicia.alvarez@ugto.mx. ORCID: https://orcid.org/0000-0001$5538-7634$

3Doctora en Ciencias de Enfermería. Docente en Campus Celaya Salvatierra. División de Ciencias de la Salud e Ingenierías Departamento de Enfermería y Obstetricia de la Universidad de Guanajuato, México. Teléfono: 052 (461) 5985922. E-mail. leticiacc_2004@yahoo.com.mx. ORCID: https://orcid.org/0000-0002-0532-4819
} 
Care in the newborn according to beliefs and customs in different cultures

ABSTRACT

Keywords:

Care

Culture

Cross-cultural nursing

Women indigenous

Newborn infants
This essay corresponds to a review of the cultural care of indigenous women's newborn infants and the contribution to cross-cultural nursing. In indigenous areas, caring for a newborn includes a world view of their beliefs, customs, traditions and norms related to health or the treatment of disease. In general, the states with the highest indigenous populations have the highest levels of maternal mortality. Knowing about cultural care makes it possible to identify the practices and beliefs that are usually thought of as retrograde traditions compared to scientific and technical development by establishing a communication bridge between the self-care that indigenous women practice. In conclusion, the birth of a child is considered an event of great social and cultural importance not only for the family, but also for the community. It can be said that it is necessary to achieve cultural competence to offer care that is culturally consistent with the needs of indigenous women in terms of care for the newborn. It is essential to obtain knowledge through research, apply the results of research to the practice of nursing care and thus move towards crosscultural nursing.

\section{Introducción}

Con respecto a los pueblos indígenas, se calcula que hay unos 370 millones de indígenas en cerca de 90 países en todo el mundo. Al practicar tradiciones singulares, mantienen unas características sociales, culturales, económicas y políticas que son distintas de las que predominan en las sociedades dominantes en las que viven 1.

El derecho a la salud en general, y para los pueblos indígenas, se encuentra regulado por muchos tratados internacionales celebrados por el gobierno mexicano. La Organización Mundial de la Salud establece que: "La salud es un estado de completo bienestar físico, mental y social, y no solamente la ausencia de afecciones o enfermedades"2; además, explica que el goce del grado máximo de salud es uno de los derechos fundamentales de toda persona y que la salud de toda la sociedad es una condición fundamental para lograr la paz y la seguridad, dicho objetivo solo puede ser alcanzado por la cooperación que se dé entre las personas y los gobiernos. De la misma manera, la Ley General de Salud reglamenta el derecho que tiene toda persona al acceso de la salud, establecido en el artículo 4o. de la Constitución Política de los Estados Unidos Mexicanos, dentro de las finalidades de este artículo es el disfrute de servicios de salud y de asistencia social que satisfagan las necesidades de la población?

Así mismo en el Artículo 24. 1. Refiere que los pueblos indígenas tienen derecho a sus propias medicinas tradicionales, manteniendo sus prácticas de salud tradicional, así como la conservación de sus plantas, animales y minerales de interés vital desde el punto de vista médico. Las personas indígenas también tienen derecho de acceso, sin discriminación, a todos los servicios de salud. Al igual que tienen derecho a disfrutar del nivel más alto posible de salud física y mental ${ }^{3}$.

Hay que mencionar además que las poblaciones indígenas tienen usos y costumbres propias, así como poseen formas particulares de comprender 
el mundo y de interactuar con él. A si mismo visten, comen, celebran sus festividades, conviven y nombran a sus propias autoridades, de acuerdo a esa concepción que tienen de la vida. Un elemento muy importante que los distingue y les da identidad, es la lengua con la que se comunican. En México, 7382785 personas de 3 años y más de edad hablan alguna lengua indígena, las más habladas son: náhuatl, Maya y Tseltal ${ }^{3}$.

Es por ello que las creencias y prácticas que se aplican en la comunidad, según sus conocimientos adquiridos se determina, a través de las costumbres de las personas, manifestadas a través de su lengua, experiencias y por sus valores, que nos llevan a conocer su entorno en relación a las prácticas culturales que han pasado de generación en generación en el cuidado del recién nacido.

En ese sentido, es importante reconocer que cada persona, cada grupo o subgrupo tienen sus propias prácticas, creencias, valores y tradiciones. No es posible pretender homogeneizar el cuidado y pensar que los de una cultura sean válidos para las demás culturas con diferentes creencias, valores y prácticas; por ello es necesario buscar, descubrir, comprender e interpretar las circunstancias del comportamiento de las personas en torno al cuidado. Cuanto más se conozca de una cultura, más fácil será predecir su conducta y las normas que esa conducta transmite a los individuos que pertenecen a ella ${ }^{4}$.

En el caso de Cuidado Cultural de Enfermería este ha emergido como un importante tópico en la Ciencia de la Enfermería, porque cuando el profesional de enfermería conoce los valores culturales, creencias y modo de vida de los individuos, el cuidado que se brinda es mucho más provechoso e integral5. Asimismo, cada individuo interioriza y aplica su cuidado según sus costumbres, sus creencias, y desde el inicio del mundo estas técnicas de alguna manera les han servido para sobrevivir y mantenerse en el tiempo; por consiguiente, los Cuidados de Enfermería deben tener presente estos postulados 6 .

De modo que, el recién nacido como resultado del proceso de gestación, es un ser cuyo desarrollo se ha realizado por diversos factores personales, familiares y sociales en las que la madre está involucrada, además de los factores como el medio ambiente y los espíritus y ritos propios de la concepción. En el presente ensayo vamos a presentar algunas costumbres $y$ creencias en torno al recién nacido desde la cosmovisión de diferentes culturas.

\section{Desarrollo}

El cuidado en torno al recién nacido desde la perspectiva cultural se constituye en un reto que lleva consigo diferentes significados en la concepción del quehacer para mantener, proteger y fortalecer la vida del niño. El conocer los cuidados culturales permite identificar $y$ significar las prácticas y creencias que suelen pensarse como tradiciones retrógradas comparadas con el avance técnico científico al establecer un puente de comunicación entre los cuidados propios que tienen las mujeres indígenas.

La enfermería transcultural tal como la define Leininger es la clave para desbloquear la competencia cultural en un entorno de salud para una nación con un pasado histórico tan rico y un futuro culturalmente diverso. Por lo que aquellos que exhiben incompetencia cultural no caben en el ámbito de cuidado de la salud 7 . En el cuidado de la salud es importante tomar en cuenta las costumbres o ideologías del paciente para evitar la aplicación de cuidados que se pueden considerar invasivos y provoquen un choque cultural, ya que esto puede provocar que la persona que necesita atención medica se aleje y por consecuencia se afecte su salud, es por ello que la enfermería debe de tomar en cuenta la interculturalidad de las personas que reciben sus cuidados. 
La profesión de enfermería cuenta con la teoría de la universalidad y diversidad del cuidado cultural, de Madeleine Leininger la cual ha definido la enfermería transcultural como un área principal de la enfermería que se centra en el estudio comparativo y el análisis de las diferentes culturas y subculturas del mundo con respecto a los valores sobre los cuidados, la expresión y las creencias de salud y la enfermedad, y el modelo de conducta, cuyo propósito consiste en concebir un saber científico y humanístico para que proporcione una práctica de cuidado de enfermería específico para la cultura y/o una práctica de cuidados enfermeros universales de la cultura ${ }^{8}$.

Desde esta perspectiva podemos decir que el recién nacido donde emergen los cuidados más susceptibles a creencias, prácticas ancestrales y mitos que influye directamente en la salud del recién nacido, el propósito del cuidado es la salud y la calidad de vida del niño, lo cual se puede lograr mediante una interacción que se ejerce entre la madre, la familia y los cuidadores; es allí donde se comparten conocimientos, experiencias y percepciones acerca del cuidado, y a su vez se generan responsabilidades para que se alcance este fin. Para lograrlo, es preciso reconocer que la práctica del cuidado difiere de un lugar a otro y de un tiempo a otro, porque el cuidar como toda actividad humana se ejecuta siempre en el seno de una determinada cultura y una sociedad, es decir, se articula con la cultura en el marco de un contexto histórico. Así el cuidado del recién nacido es vía de transmisión, conservación y actualización de la cultura de una generación a otra.

Respecto a las prácticas, creencias culturales en el cuidado del recién nacido en determinadas culturas pueden afectar sus actitudes, estas creencias pueden variar dentro de grupos particulares.

A continuación, se mencionarán algunas creencias culturales en el cuidado del recién nacido ${ }^{9}$ :
Prácticas en el cuidado

del recién nacido

Creen que el calostro es dañino (viejo, rancio, sucio, venenoso o

Estadounidenses de contaminado) para él origen asiático bebé, por lo que pueden demorar la lactancia materna hasta la llegada de la leche.

Posponen la lactancia ya que consideran el

Estadounidenses calostro dañino y sucio. nativos

Utilizan mochilas para bebes y no manipulan mucho al bebe.

Demoran la lactancia debido a que el calostro es considerado sucio $y$ podrido.

Usan un collar religioso que se coloca alrededor

Hispanoamericanos del cuello del neonato tras el nacimiento.

No circuncidan a los lactantes varones.

Pueden colocar una faja abdominal al recién nacido para evitar la hernia umbilical.

Ayunan durante el

Arabeamericanos embarazo para tener un hijo varón.

Ven el amamantamiento como algo vergonzoso $y$, por lo tanto, alimentan con biberón.

Afroamericanos Introducen alimentos sólidos tempranamente Colocan aceite en la piel del bebe.

Fuente: Stephanie C. Butkus ${ }^{9}$

Consideremos ahora en una tesis Doctoral donde, Machado R, Valerio $\mathrm{S}^{10}$. En Nicaragua, refieren que al momento que se da la caída del cordón umbilical, la madre lo guarda porque sirve 
para remedio (hierven el ombligo en agua de azúcar y dan esta bebida al niño cuando creen que va a enfermar). El enterrar el cordón umbilical a la entrada de la casa o al pie de un árbol cercano, evita que el muchacho salga andariego. La población campesina cree también que la gente no olvida el lugar donde tiene enterrado el ombligo, y que, aunque se marche lejos, algún día retornará10. Es importante el poder respetar como personal de salud esta costumbre que no interfiere con la salud del recién nacido al contrario muestra sus creencias y costumbres arraigadas.

Ahora bien, durante los 8 días posteriores al parto, la partera encomienda al papá matar un gallito si el recién nacido fue niño y una pollita si fue niña, ambos animales que no pesen más de un kilo; lo hacen a manera de ofrenda para evitar hemorragias y complicaciones 10 .

En el caso de la cosmovisión Awajún Wampis en el Perú, en un estudio en la selva amazónica, menciona Amaro W. ${ }^{11} q u e$ inmediatamente después del nacimiento, el recién nacido es bañado con agua y plantas medicinales como el piripiri, a fin de protegerlo contra diversos males como el cutipado (susto). Es importante que la madre cuide al niño durante los primeros días para que no se "cutipe", es decir no lo dé el susto y los espíritus le generen daño, ya que esto podría causarle en el peor de los casos la muerte, en esta tarea la madre recibe el apoyo del esposo. De acuerdo con la concepción Awuajun y wampis una de las causas para que se dé el cútipado del niño es el hecho de que la madre o el padre salgan al bosque y se encuentren con animales como el zorro, el tigrillo, el paucar, el lagarto o la serpiente; por ello tanto la mujer como el hombre no salen al monte los primeros días de nacimiento del niño debido a este riesgo. Es por eso que se les hace énfasis en que el papá no debe salir al monte, ya que hay la creencia de que este puede encontrarse con algunos animales y ello puede hacer daño al recién nacido cutipándose (se enferme del susto), esto favorece la presencia del padre en la atención de la mujer en este periodo ${ }^{11}$. Esto es importante ya que aquí se le integra de esos cuidados al padre y también este inmerso en el rol y cuidado del recién nacido con sus costumbres.

En un estudio en la comunidad nativa Camisea del pueblo Matsigenka, Cusco, Perú refieren que antes para que los bebés crecieran sanos no podían salir de la casa al menos tres meses, limitando que estos fueran vistos por otras personas que no sean los parientes cercanos. $\mathrm{Ni}$ ver la luna llena porque según las historias, la luna se lo puede llevar y comérselo. Es interesante conocer la cosmovisión de cada pueblo para poder entender y dar ese cuidado cultural que se manifiesta ${ }^{12}$.

En un estudio comparativo de la cultura marroquí y valenciana se encontró lo siguiente con relación al cordón umbilical, se lo guardan en ambos grupos, como tradición en España, desconociendo el significado de porqué lo hacen y como amuleto de la suerte en Marruecos. En ambas culturas se constata el nacimiento preferencial de varón (como garante de la continuación de la herencia patrilineal la marroquí, con el fin de que no se pierdan los apellidos del clan patrilineal la española). Los cuidados del recién nacido en ambos grupos culturales los podríamos resumir en los dispensados a los ojos, el ombligo o la piel del recién nacido y en esto no se diferencian ambos grupos culturales. Las diferencias vienen dadas por los productos empleados y la forma de aplicarlos, en Marruecos, subsisten pautas culturales muy ancladas en la tradición en el cuidado infantil, como el empleo de khol y henna, con una finalidad mágica y ritual de protección. El khol se emplea para el maquillaje de ojos o cejas y la henna en la piel, el pelo y el ombligo. Mantienen las mujeres marroquís por tradición, el enfajado completo del bebé (ssmat) durante unas semanas. Todas las tareas relacionadas con el cuidado de los bebés están en manos de mujeres. Se realiza un ritual que consagra al recién nacido como miembro de la comunidad religiosa y todo esto se celebra con una comida 
para familia y amigos. La circuncisión (al jitan) en Marruecos es obligatoria, en España no se realiza. La transmisión de la cultura en el entorno al nacimiento es oral en ambas culturas, en el ámbito familiar ${ }^{3}$.

Por los argumentos señalados se deduce que la meta de la enfermería transcultural es proporcionar cuidados que sean coherentes con los valores, las creencias y las prácticas culturales. Dado que las culturas muestran tanto diversidad (perciben, conocen y practican los cuidados de formas diferentes) como universalidad (características comunes de los cuidados).

Es importante señalar que algunas de las principales características de la Enfermería transcultural son: Vincula a la enfermería con la diversidad cultural y se presenta como una herramienta práctica y útil para mejorar la comprensión de los profesionales ante las creencias y problemas relacionados con la salud de personas procedentes de diferentes culturas ${ }^{8}$.

En concreto el nacimiento de un niño es considerado un acontecimiento de gran importancia social y cultural no tan sólo para la familia, sino también para la comunidad. En palabras de Leininger ${ }^{14}$ comprender las creencias y prácticas de cuidado que las madres brindan a sus hijos recién nacidos contribuye a la profundización del conocimiento en torno a la diversidad cultural inmersa en ese modo particular de cuidar.

\section{Conclusiones}

Desde la perspectiva cultural se constituye en un reto que lleva consigo diferentes significados en la concepción del quehacer para mantener, proteger y fortalecer la vida del niño.

En el cuidado de la salud es importante tomar en cuenta las costumbres o ideologías de las mujeres indígenas y sus familias, para evitar la aplicación de cuidados que se pueden considerar invasivos y provoquen un choque cultural, ya que esto puede ocasionar que la persona que necesita atención medica se aleje y por consecuencia afecte su salud, es por ello que la enfermería debe de tomar en cuenta la interculturalidad de las personas que reciben sus cuidados.

Ya que el conocimiento es el primer paso hacia la incorporación del cuidado cultural en la práctica de enfermería diaria en la atención al cuidado del recién nacido.

Se surgiere que los profesionales de enfermería tengan descripciones breves de las culturas, así como sus puntos de vista sobre la salud, enfermedad, la alimentación entre otros temas.

Lo anterior exige al profesional de la salud indagar el conocimiento acerca de los cuidados culturales en cada región donde se encuentren para poder trasculturalizar y ofrecer un cuidado holístico dentro de la cultura de cada familia, se puede decir que es necesario alcanzar competencia cultural para ofrecer cuidado culturalmente congruente a las necesidades de estas mujeres y sus recién nacidos. Es indispensable obtener conocimientos por medio de la investigación, llevar los productos de la investigación a la práctica del cuidado de enfermería y así avanzar hacia la enfermería transcultural.

\section{Bibliografía}

1. Organización Naciones Unidas. Pueblos indígenas. ONU; 2015, Disponible en: https://www.un.org/es/events/indigenousda $\mathrm{y} / 2015 / 2015$ theme.shtml

2. Organización Mundial de la Salud (OMS). Página oficial de la Oficial de la Organización Mundial de la Salud [Internet]. Suiza (Berna): Oficina Sede para la Organización Mundial de la Salud; 2020; consultado 25 de mayo 2020]. Disponible en: http://origin.who.int/about/mission/es/

3. El derecho a la salud de los pueblos indígenas. Segunda edición. Comisión Nacional de los Derechos Humanos: México: 2015. 21 p. 
4. Palacios D, Salvadores P. Enfermería Social. España. Editorial Síntesis, S.A; 2012. 206 páginas.

5. Castillo J. El cuidado cultural de enfermería: necesidad y relevancia. Rev. haban cienc méd [Internet]. 2008 Sep [citado 2020 mayo 22]; 7(3): Disponible en: http://scielo.sld.cu/scielo.php?script=sci_abs tract\&pid $=$ S1729519X2008000300003\&lng =es

6. Fernández MF. La enfermería en la historia: un análisis desde la perspectiva profesional. Cultura de los cuidados. (Internet) España: 2003. [Acceso 19 de mayo de 2020].1 (2) 35 páginas. Disponible en: http://www.academia.edu/3244322/La_enfer mer\%C3\%ADa_en_la_historia_Un_an\%C3\%A1 lis is_desde_la_perspectiva_profesional.

7. Busher D. "Madeleine Leininger and the Transcultural Theory of Nursing." The Downtown Review. [internet]. 2016 [consultado el 30 septiembre 2017]; 2(1): 1 Disponible en: http://engagedscholarship.csuohio.edu/tdr/v ol $2 /$ iss $1 / 1 /$.

8. Moreno M., Muñoz L. De la teoría de enfermería a la práctica. Editoras científicas, Universidad de La Sabana, Bogotá Colombia. 2016. 372 p.

9. Stephanie C. Butkus. Enfermería MaternoNeonatal. 3ed. España. Editora Clínica. Copyright de la edición en español (c) 2016 Wolters Kluwer. 598p.

10. Machado R, Valerio S, "Creencias, mitos y prácticas que tienen las mujeres sobre el embarazo, parto y puerperio en el Centro de Salud Sócrates Flores Vivas, enero a marzo 2016" (tesis doctoral). (Nicaragua): Universidad Nacional Autónoma de Nicaragua Managua; 2017. 82p.

11. Amaro W. Cosmovisión Awajún- Wampis en la Salud y Enfermedad. Perú. Indecopi, 2017, 144 páginas.

12. Araujo Salas B. Costumbres en torno al parto en la comunidad nativa Camisea del pueblo Matsigenka, Cusco, Perú. IS [Internet]. 30may2018 [citado 27may2020];21 (39):27-0.
Available

from: https://revistasinvestigacion.unmsm.edu.pe/ index.php/sociales / article/view/ 14659

13. Moreno C, Vidal. Cultura del nacimiento: transmisión oral de los cuidados y creencias tradicionales. (estudio comparativo de la cultura marroquí y valenciana) (tesis doctoral). (Valencia, España): Universidad Católica de Valencia San Vicente Mártir; 2016. 374p.

14. Leininger $M$. Culture Care Diversity and Universality: a Theory of nursing. (libro online). 1991. Disponible en: https://archive.org/details/culturecarediver0 Olein/page/n7/mode/2 up 